\title{
Tolosa Hunt syndrome involving the pituitary: A Case
}

\section{report}

\section{Sefika Burcak Polat ${ }^{1}$, Oya Topaloglu, ${ }^{1}$ Ercan Bal $^{2}$, Reyhan Ersoy $^{1}$, Bekir Cakir $^{1}$}

${ }^{1}$ Yildirim Beyazit University, Faculty of Medicine, Department of Endocrinolocx gy and Metabolism, Ankara, Turkey

${ }^{2}$ Yildirim Beyazit University, Faculty of medicine,Neurosurgery Department, Ankara, Turkey

Introduction

Tolosa Hunt syndrome (THS) is described as unilateral orbital pain associated with paresis of one or more of the third, fourth and/or sixth cranial nerves caused by a granulomatous inflammation in the cavernous sinus, superior orbital fissure or orbit. Pituitary gland is rarely involved in this syndrome. Herein we represented a case with hypophysitis with Tolosa Hunt who was operated with the suspicion of pituitary adenoma.

\section{Case}

$>$ A 63-year-old female patient with diabetes history admitted to our clinics with the complaints of weight gain and headache. In the physical examination, BMI was $40 \mathrm{~kg} / \mathrm{m}^{2}$ and there was buffalo hump, central obesity and acanthosis nigricans. With the suspicion of Cushing syndrome, biochemical screening was performed with low dose dexamethasone suppression test and free cortisol in 24-hour-urine collection. Serum cortisol after 1 mg dexamethasone suppression was 2.2 whereas free urinary cortisol was normal. For exclusion of pseudocushing, we performed 2 days $2 \mathrm{mg}$ dexamethasone suppression with $\mathrm{CRH}$ and the result was equivocal (cortisol after CRH: 1.5). Her ACTH value was 20, and high dose dexamethasone test result was consistent with Cushing's disease. In the CRH test there was $\% 300$ increase in $\mathrm{ACTH}$ and \%100 increase in cortisol after the administration of CRH. In the pituitary MRI, a lesion $1.8 \mathrm{~cm}$ in size was reported. While the evaluation was going on, the severity of headache was increased and ophtalmoplegia developed. The patient underwent endoscopic transsphenoidal surgery. The histopathology of the lesion was consistent with granulamatous reaction with caseous necrosis. Tuberculosis and sarcoidosis was excluded by PPD, thorax CT, lumbar puncture and analysis of the cerebrospinal fluid, mycobacterium tuberculosis PCR and the culture of the pathology specimen. The final diagnosis was Tolosa Hunt

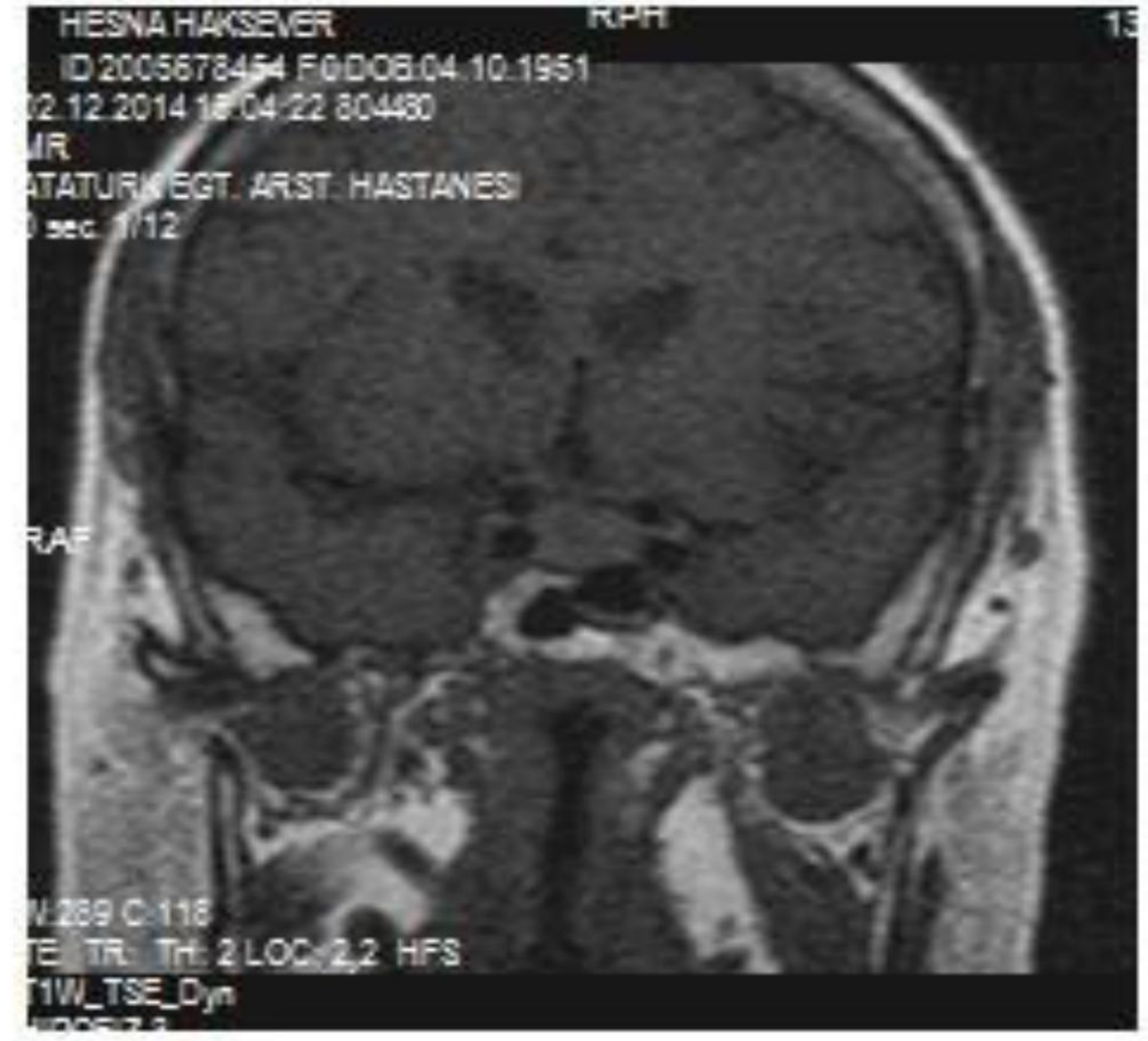

Figure 1. Pituitary gland is enlarged with heterogenous appearnce

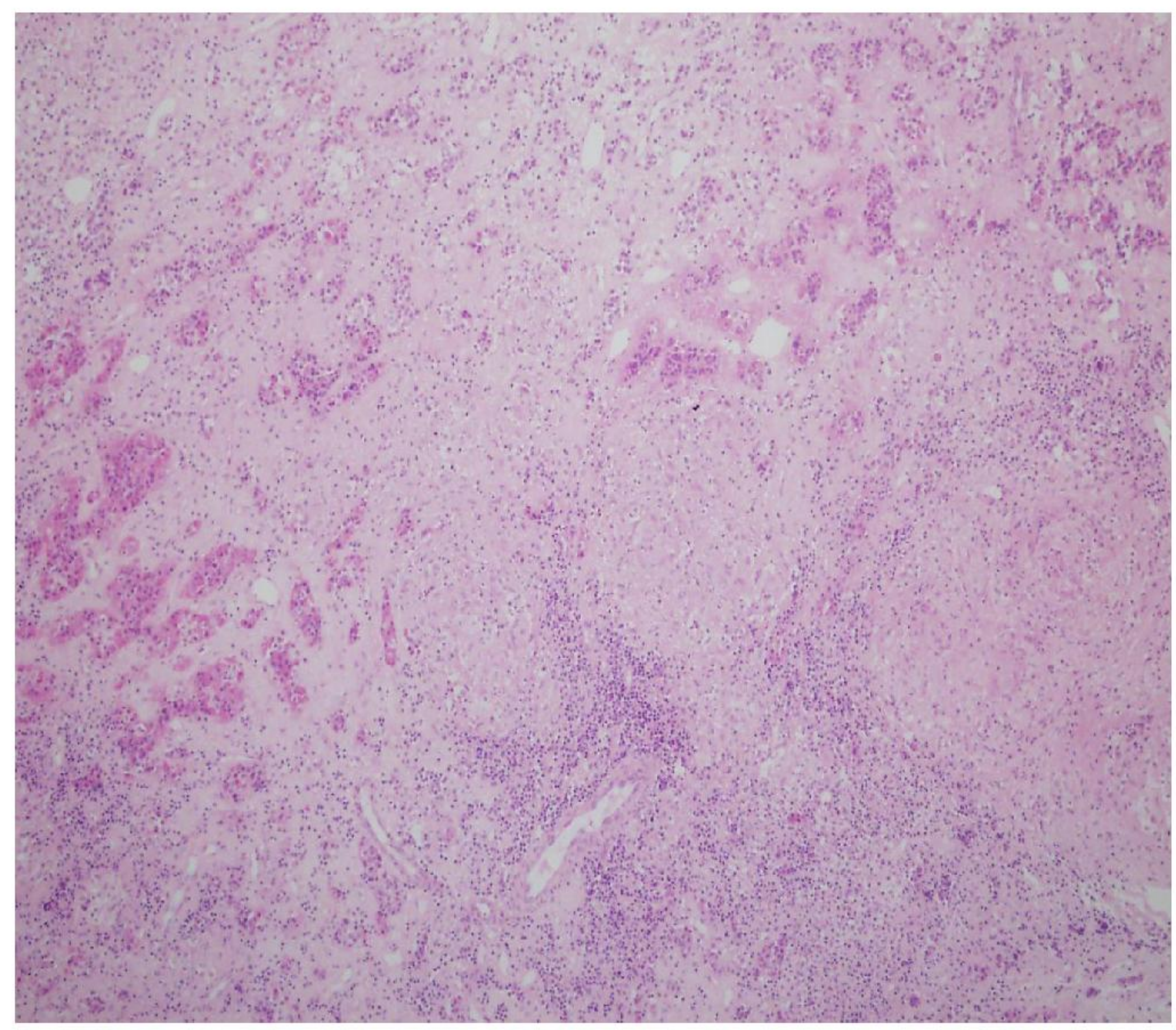

Figure 2: Granulamtous inflammation with in the pituitary gland

Conclusion: Tolosa Hunt is a very rare cause of hypophysitis and panhypopituitarism. It should be considered in the differential diagnosis of a sellar mass. 